\title{
Implante quirúrgico de radiotransmisores en Diplomystes camposensis (Siluriformes, Diplomystidae)
}

\author{
Surgical implantation of radio transmitters in Diplomystes camposensis \\ (Siluriformes, Diplomystidae) \\ K Solis-Lufi*, A Jara, N Colin, J González, A Oyanedel, E Habit \\ Centro de Ciencias Ambientales EULA-Chile Universidad de Concepción, Concepción, Chile.
}

\begin{abstract}
SUMMARY
Surgically implanted radio transmitters are useful for monitoring fish behaviour, movement patterns and home range. In Chile, there are no reports using this particular technique in native freshwater fish. Therefore, no standardized protocols have been developed in Chile to describe surgical procedures for inserting the implant nor the required dose of anaesthetics and medications applied to avoid fish mortality. This study describes a procedure for implanting a radio transmitter in Diplomystes camposensis, a native fish of southern Chile, which can be valuable for future research. The procedure was successful on these organisms because it prevented post surgical mortality and allowed fish monitoring throughout the entire life span of the transmitter. Our research can serve as a guide for future studies involving surgical implants of radio transmitters on native fish with high conservation values, in which mortality due to research should be minimal.
\end{abstract}

Palabras clave: anestesia, radiotransmisores, protocolo, Diplomystes camposensis.

Key words: anaesthesia, radio transmitter, protocol, Diplomystes camposensis.

\section{INTRODUCCIÓN}

Diplomystes camposensis es un pez nativo perteneciente a la familia Diplomystidae, grupo basal del orden Siluriformes o bagres (Arratia 1987, de Pinna 1998). Diplomystes camposensis presenta distribución restringida a la cuenca hidrográfica del río Valdivia (Arratia 1987, Habit y col 2008). Actualmente es considerada como una especie en peligro de extinción ${ }^{1}$. La biología de esta especie es prácticamente desconocida, pero por su semejanza morfológica se ha postulado que no diferiría de los otros diplomístidos (Ruiz y Marchant 2004). Para las especies congenéricas $D$. chilensis y $D$. nahuelbutaensis se han descrito cambios ontogenéticos en el uso del hábitat, en que los adultos utilizan ambientes de mayor velocidad y profundidad que los juveniles (Arratia 1983, Habit 2005). De igual manera, Vila y col (1996) señalan para D. nahuelbutaensis una baja fecundidad, con un máximo de 330 oocitos maduros y un desove que tiene lugar concordantemente con los caudales más bajos del año (fines de verano y comienzos de otoño). La máxima longitud conocida para esta especie en su estado adulto es de $249 \mathrm{~mm}$ (Arratia 1987). De acuerdo a estudios recientes la mayor población de esta especie habita en la

Aceptado: 22.04.2009.

* Centro EULA-Chile, Universidad de Concepción. Casilla 160-c, Concepción, Chile; katherin.solis@gmail.com

1 CONAMA 2007. http://www.conama.cl/clasificacionespecies/ Anexo_tercer_proceso/Diplomystes_camposensis.doc consultado el 20 de junio de 2008 zona de encajonamiento del río San Pedro, tributario de la cuenca del Valdivia (Habit y col 2008).

Muchos aspectos de la biología de la fauna íctica nativa de aguas continentales de Chile, tales como patrones de desplazamientos, migración y comportamiento, son completamente desconocidos. Una metodología robusta, que constituye una herramienta común en el estudio de peces (Mulcahy 2003) y que permite responder a las interrogantes antes mencionadas, es el seguimiento continuo de individuos a través de radiotelemetría, siendo la mejor alternativa el uso de transmisores implantados en la cavidad celómica. Sin embargo, esta metodología tiene algunas limitantes para los peces nativos chilenos, los cuales se caracterizan por ser de pequeño tamaño (Vila y col 1999), por lo que no ha sido utilizada previamente en Chile.

Actualmente, existe un importante cuerpo de conocimiento sobre biología de peces utilizando telemetría (Jepsen y col 2002), así como sobre los mejores métodos de marcaje y sus usos (Stasko y Pincock 1977, Baras 1991, Nielsen 1992). Tal información se encuentra enfocada principalmente a cómo interpretar y procesar los datos obtenidos. Sin embargo, la literatura concerniente con el mejoramiento de la técnica de implantación de radiotransmisores en diferentes especies de peces es escasa (Summerfelt y Smith 1990, Baras y col 2003). La información que se posee hoy en día al respecto es que este tipo de procedimiento puede causar variados efectos subletales (Mulcahy 2003) y efectos secundarios, los que incluyen la disminución de la supervivencia (Manns y Whiteside 1979), comportamiento alterado (Diana 1980, Manns y Whiteside 1979, 
Mesing y Wicker 1986, Thoreau y Baras 1997), pérdida de la flotabilidad (Gallepp y Magnuson 1972), infecciones (Paukert y col 2001), pérdida del equilibrio (Thoreau y Baras 1997), reducción de la alimentación (Lucas 1989, Mortensen 1990, Thoreau y Baras 1997) y aumento de la depredación (Adams y col 1998). Sin embargo, tal como señalan Baras y col (2003), la mayoría de los efectos nocivos en comportamiento y fisiología de los peces, producto del marcaje con procedimientos quirúrgicos, pueden ser aliviados o suprimidos adaptando el procedimiento de la cirugía, el equipo y el cuidado operativo. Generalmente, éstas son recomendaciones muy generales, por lo tanto, con un limitado valor para ser replicadas (Mulcahy 2003). Ello se debe principalmente a que no existen protocolos de laboratorio estandarizados que describan la metodología de implante mediante cirugía y el uso adecuado de anestésicos y soluciones tópicas e intraperitoneales de asepsia que aseguren una mayor probabilidad de sobrevivencia de los individuos implantados. Esto resulta de vital importancia para especies como D. camposensis, la cual es clasificada como especie rara, y por ende la mortalidad debida a estudios científicos debe ser reducida al mínimo.

De esta manera, este trabajo tiene como objetivo describir en detalle el protocolo experimental para el implante de radiotransmisores en trece individuos de $D$. camposensis, por lo que pretende servir de guía para futuras investigaciones de cirugías enfocadas a conocer aspectos biológicos de peces nativos, especialmente de aquellas especies de importancia para la conservación.

\section{MATERIAL Y MÉTODOS}

Durante el periodo comprendido entre marzo 2006 a febrero 2007 se realizaron muestreos diarios de peces en las zonas ribereñas y pozones del río San Pedro, cuenca del Valdivia, desde el sector del Desagüe del Lago Riñihue $\left(39^{\circ} 46^{\prime} \mathrm{S} ; 7^{\circ} 27^{\prime} \mathrm{W}\right)$ hasta $500 \mathrm{~m}$ aguas arriba de la confluencia con el río Quinchilca (39 $51^{\prime}$ S; $72^{\circ} 27^{\prime} \mathrm{W}$ ).

Fueron capturados trece individuos mediante pesca eléctrica (equipo Smith Root LR-24) y anzuelos con carnada viva en campañas de pesca nocturna. En terreno, cada individuo fue anestesiado mediante solución de benzocaína anestésico-tranquilizante para peces (BZ-20 ${ }^{\circledR}$ ) en solución $1 \mathrm{ml}: 5 \mathrm{~L}$, medido (longitud estándar con ictiómetro de $0,1 \mathrm{~cm}$ de precisión) y pesado (peso húmedo mediante balanza digital Ohaus modelo Scout pro, de 400 o $2000 \mathrm{~g}$ dependiendo del peso del pez) para reducir su estrés por manipulación. Posteriormente, los ejemplares fueron transportados al laboratorio en estanques de $50 \mathrm{~L}$ con oxigenación continua.

Una vez en el laboratorio, los peces se mantuvieron en una etapa de aclimatación en acuarios de 500 L desinfectados previamente con Duplalim ${ }^{\circledR}$ (Veterquímica) en solución 1:200, al menos durante 24 horas antes del implante, sin ser alimentados para maximizar el espacio donde se implantó el radiotransmisor en la cavidad celó- mica y permitir una mayor capacidad de manipulación quirúrgica. El procedimiento de implante que se describe en este trabajo se basa en la microcirugía exitosa de 13 ejemplares de $D$. camposensis.

Debido a que uno de los puntos de mayor importancia en la implantación de un radiotransmisor es que éste no interfiera en la capacidad de nado del pez, se siguieron las recomendaciones de los fabricantes para establecer tanto el peso mínimo de un pez a ser implantado como el tipo de radiotransmisor a utilizar. Se consideró que el peso del radiotransmisor no debía superar el $2 \%$ del peso corporal del pez (Schulz y Leuchtenberger 2006, Schulz 2003, Winter 1996), por tanto, como los peces capturados pesaron entre 40 y $140 \mathrm{~g}$ se utilizaron radiotransmisores de la serie de nanotag de Lotek modelo NTC-6-1, de 2,8 g de peso, vida útil de 124 días y sus dimensiones son $22,4 \mathrm{~mm}$ de longitud-9, 1 de diámetro y NTC-4-2L de 2,1 $\mathrm{g}$ de peso, vida útil de 55 días y sus dimensiones son $18,3 \mathrm{~mm}$ de longitud-8,3 de diámetro.

\section{RESULTADOS Y DISCUSIÓN}

\section{PROTOCOLO DE IMPLANTE DE RADIOTRANSMISORES}

Equipo de trabajo. El grupo de trabajo estuvo conformado por un número mínimo de tres personas, equipadas con indumentaria hospitalaria, quienes cumplen las siguientes funciones: a) Cirujano: encargado de realizar la cirugía; b) Arsenalero: encargado de asistir al cirujano, mantener el instrumental esterilizado y los insumos necesarios para la realización del procedimiento; c) Anestesista: su función es mantener la totalidad del cuerpo del pez húmedo y anestesiado durante la cirugía, además de asegurar visualmente la respiración del pez.

Implementos de trabajo. Los implementos de trabajo se dividen en las siguientes categorías:

-Mesón de cirugía: se divide en cuatro áreas: "área limpia", "área sucia", "área de procedimiento" y "área de anestesia", logrando de esta manera orden en la utilización y disposición del instrumental de trabajo y la minimización de contaminación por agentes patógenos, que pudiesen ocasionar contaminación cruzada o infección durante el procedimiento. El área limpia es el lugar donde se encuentra el instrumental y los insumos debidamente esterilizados, el radiotransmisor y el pez, y es vital para reducir la probabilidad de infecciones. El área sucia es donde se deposita el instrumental e insumos ya utilizados. $\mathrm{El}$ área de procedimiento es el lugar donde se realiza la cirugía, que contiene la camilla de cirugía. El área de anestesia es el lugar en que se almacena la anestesia (Etil p-aminobenzoato), en solución de agua estéril a razón de $1 \mathrm{ml}: 5 \mathrm{~L}$, para ser utilizada durante la cirugía. Todas las áreas deben ser desinfectadas previamente con Duplalim ${ }^{\circledR}$ (Veterquímica) en solución 1:200. 
-Camilla de cirugía: corresponde a un recipiente en el que se adapta una esponja de espuma densa con un corte en forma de $\mathrm{V}$ en el centro, donde se ubica el pez. Este corte debe sostener el cuerpo del pez de tal forma de dejarlo inmovilizado, por lo que es específico para cada individuo. Se requiere de mayor profundidad y diámetro para sumergir la cabeza y branquias, lo que facilita la respiración del pez durante la operación. Esta zona debe mantenerse constantemente con anestesia en solución para mantener al pez vivo y anestesiado durante el procedimiento. Previo al procedimiento, la camilla debe ser desinfectada con solución de Duplalim ${ }^{\circledR}$ (Veterquímica) 1:200, y saturada en agua estéril para mantener la humedad del cuerpo del pez.

-Instrumental quirúrgico (equipamiento mínimo): para el implante de un radiotransmisor se requiere de un conjunto mínimo de instrumentos quirúrgicos metálicos esterilizados compuestos por pinzas finas, bisturíes, pinzas gruesas, tijeras quirúrgicas agudas, cajas metálicas y portaagujas mayo. Además se utilizó un recipiente para anestesia, dosificador de anestesia (recipiente pequeño con el cual se agregó anestésico a la camilla de cirugía durante el implante, en cantidades menores), aspersores (para alcohol y agua filtrada) e iluminación adecuada mediante lámpara de pie articulado. El instrumental plástico fue desinfectado con Duplalim ${ }^{\circledR}$ (Veterquímica) 1:200.

-Insumos quirúrgicos (equipamiento mínimo): para el implante de un radiotransmisor es necesario contar con los siguientes implementos: agujas de 18G X 1 1/2" (40X12), hojas de bisturí $10 \mathrm{~N}^{\circ} 3$ (Fitment), agujas curvas para sutura con ojo resorte estilo 508 tamaño 23 (Fadimed) y sutura quirúrgica absorbible (monofilamento Cat Gut N 4), 5 L de solución de Sol-Flox ${ }^{\circledR}$ 0,15:1.000, alcohol etílico anhidro $99,3^{\circ}$, povidona yodada en solución tópica al 10\%, anestesia BZ-20® en solución de agua estéril en razón de $1 \mathrm{ml}: 5 \mathrm{~L}$, varillas de algodón, papel absorbente y cubierta plástica para camilla tipo Steri-Drape de $35 \mathrm{~cm} \times 35 \mathrm{~cm}$. Cada cirugía debe utilizar instrumentos quirúrgicos estériles para evitar la contaminación cruzada (Mulcahy 2003).

-Estanque de recuperación: estanque oscuro en el que el pez se mantiene durante el proceso de recuperación postoperatorio. Previo a su uso debe ser desinfectado con Duplalim ${ }^{\circledR}$ 1:200 y mantenido con agua corriente durante todo el proceso de recuperación.

-Estanque de tratamiento: estanque o cubeta con aireación de menor capacidad que el de recuperación, en el cual se aplica un baño de antibiótico Sol-Flox ${ }^{\circledR}$ 0,15:1.000.

Cirugía. El procedimiento de implante del radiotransmisor comienza con la sedación del pez, y termina con su ingreso al estanque de recuperación. Esta etapa dura un promedio de 40 minutos aproximadamente.

-Preparación inicial (Paso 1): Previo al inicio de la cirugía se desinfectan las superficies que estarán en contacto con el pez. Se prepara la anestesia (BZ-20®) en un recipiente de $20 \mathrm{~L}$ y se cubre la camilla con su capa. Una vez preparado el instrumental, el equipo de trabajo debe renovar sus guantes quirúrgicos y utilizar mascarilla.

-Aplicación de anestesia (Paso 2): El anestesista sumerge al pez en un recipiente con solución de anestesia y lo mantiene en observación hasta que es posible manipularlo sin forzarlo con la zona ventral hacia arriba. Ello es simultáneo a la reducción del ritmo de respiración (movimiento opercular mínimo) y a la ausencia de respuestas a estímulos externos. Es importante destacar que no existe una dosis única de anestesia, ya que se debe tener en consideración la especie y talla del individuo, según lo cual presentará mayor o menor resistencia a la anestesia. Además, la inducción y tiempo de recuperación es dependiente de la temperatura y debiera ser cercana a la temperatura de las condiciones naturales del hábitat del pez para evitar un estrés térmico (Jepsen y col 2002). La mortalidad es más baja cuando se renueva la solución anestésica, es decir, al suministrar agua filtrada sin anestesia y cuando el pez se encuentra completamente anestesiado mientras se realiza la cirugía (Jepsen y col 2002).

- Traslado a camilla de cirugía y ubicación del equipo de trabajo (Paso 3): El anestesista traslada el individuo a la camilla de cirugía, dejando la cabeza completamente sumergida en solución de anestesia y el cuerpo inmovilizado por el corte en la esponja. Desde este momento y hasta el traslado del pez al estanque de recuperación, el anestesista se encarga de mantener el cuerpo del pez húmedo y las branquias sumergidas en solución de anestesia. La ubicación sugerida del anestesista es al frente del cirujano. El arsenalero se ubica al lado izquierdo del cirujano, en frente del área limpia. El cirujano se ubica en frente del área de procedimiento y con el área sucia a su izquierda.

-Incisión (Paso 4): La zona de incisión se limpia mediante aspersión de agua destilada, se seca con varillas de algodón y se desinfecta con povidona yodada. Se vuelve a secar y se levanta el tejido de la pared corporal para realizar una incisión de 15 a $25 \mathrm{~mm}$ aproximadamente (dependiendo del tamaño del radiotransmisor a implantar) hasta llegar a la cavidad celómica. La incisión se realiza desplazada hacia un costado, de manera longitudinal al cuerpo del pez (figura 1).

-Implante (Paso 5): Por la incisión inicial se introduce una de las paletas de la pinza gruesa (figura 2). Una vez dentro de la cavidad celómica y manteniendo el contacto interno con la piel, se desplaza la pinza hasta la zona 


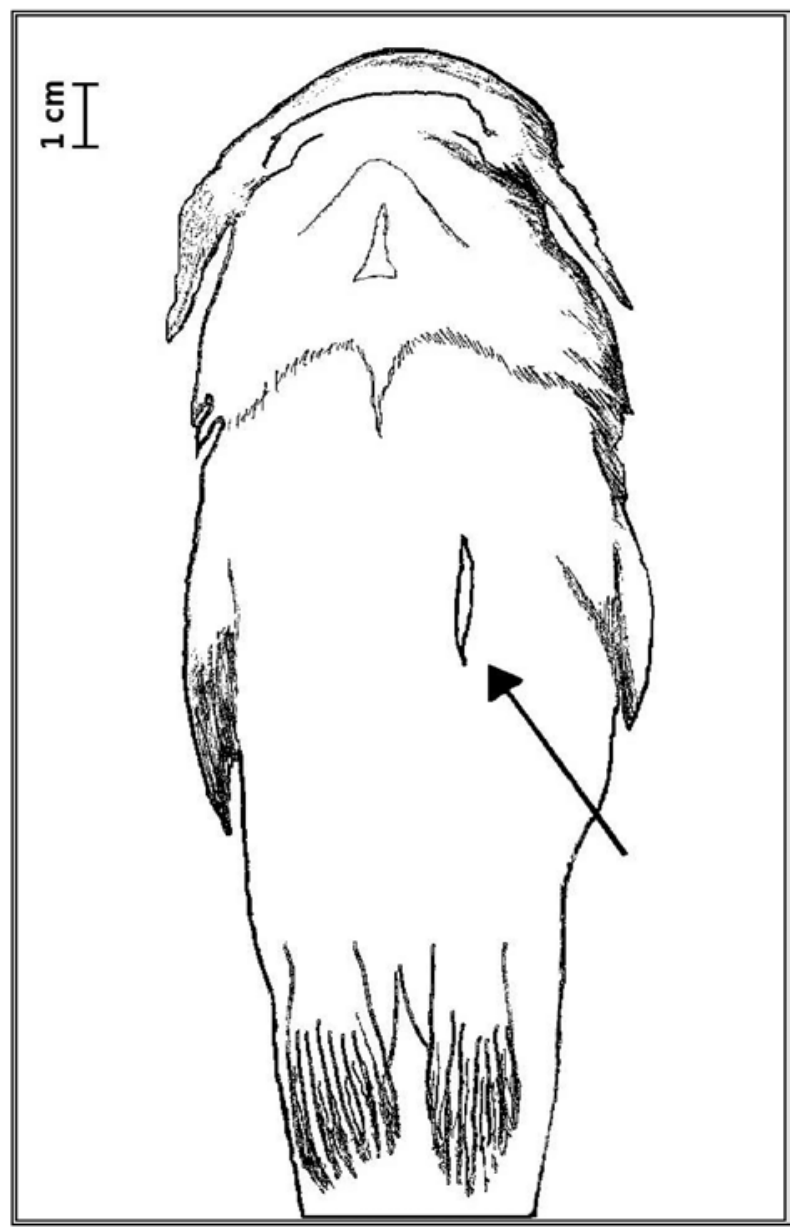

Figura 1. Ubicación y tamaño relativo de la incisión inicial en el pez.

Location and relative size of the initial incision on the fish.

lateral por donde saldrá la antena. Ubicada esta zona, se realiza en ella una segunda incisión más pequeña, insertando una aguja 18G. En este procedimiento se emplea la pinza como guía para que la aguja insertada salga por la primera incisión, sin dañar órganos internos. Luego, la antena del radiotransmisor se pasa por completo por el lumen de la aguja de 18G. Una vez introducida la antena se saca la aguja y se aloja el radiotransmisor en la cavidad celómica, el cual se ingresa por la incisión inicial. Este procedimiento concuerda con lo sugerido por Mulcahy (2003), en relación a que el transmisor se debe colocar en el celoma de una forma que evite el contacto directo con la incisión. Ésta debe ser pequeña, ya que la presencia del transmisor puede aumentar la presión y causar inflamación, lo cual contribuye a la apertura espontánea de la incisión y la pérdida del transmisor (Kaseloo y col 1992, Schramm y Black 1984, Wagner y Stevens 2000).

-Sutura (Paso 6): Generalmente en las suturas de las incisiones quirúrgicas en peces se utiliza un patrón interrumpido simple; sin embargo, un patrón continuo simple de sutura puede ser preferible por las siguientes

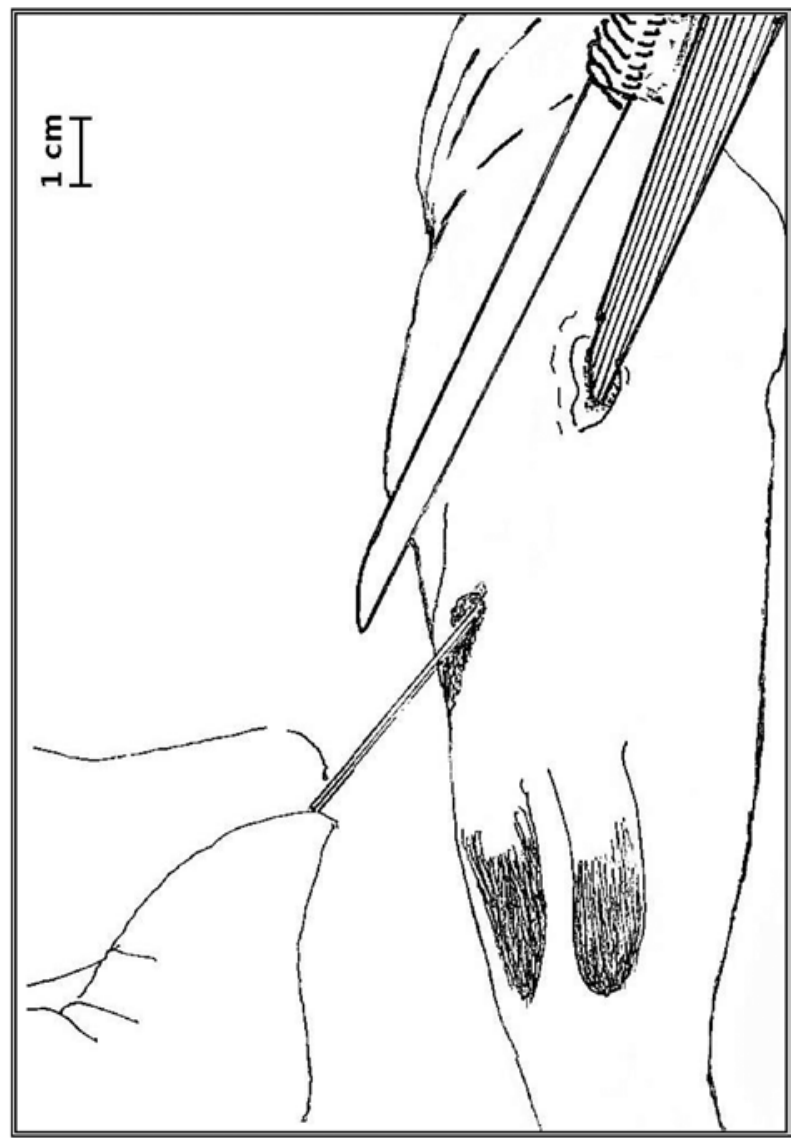

Figura 2. Procedimiento para la inserción de la antena en la segunda incisión en el pez.

Procedure for the introduction of the antenna in the second incision on the fish.

razones: deja pocos nudos y extremos de la sutura que podrían causar irritación, produce menos área superficial en la cual las bacterias se puedan fijar y absorber y es más rápida de desplegar una vez que se adquieren las habilidades (Mulcahy 2003). A continuación, se describe el procedimiento de sutura de patrón continuo simple o en cierre (tipo Reverdin) utilizado en $D$. camposensis: Con una pinza fina se levanta levemente un borde de la incisión y se inserta con un portaagujas mayo la aguja curva hilvanada con sutura quirúrgica absorbible desde afuera hacia adentro de la piel. Luego se toma el lado opuesto de la incisión y se inserta la aguja en sentido contrario. En el lugar donde se inserta al inicio la aguja se realiza un primer nudo. Es aconsejable utilizar como tope sobre la incisión que se está cerrando la punta de una pinza pequeña. Este procedimiento se repite para realizar el segundo punto. Este engancha con el punto anterior pasando por abajo del hilo (patrón Reverdin) y se continúa de la misma manera si fuera necesario realizar un tercer o cuarto punto. Una vez terminado, se realiza un nudo de cierre en el último punto. Un factor importante en esta etapa es el uso de una aguja curva de tamaño 
apropiado. Durante el tiempo del estudio se utilizaron diversos tipos de agujas existentes en el mercado, la más adecuada resultó ser la aguja tipo 508 tamaño 23 y con sutura quirúrgica absorbible simple (monofilamento Cat Gut N 4). Un número superior a éste produce trastornos en la piel, generando heridas, lo que conlleva a posibles infecciones, incremento del periodo de cicatrización y un consecuente aumento del tiempo de permanencia del pez en el laboratorio. En este sentido nuestros resultados fueron mejores utilizando este tipo de sutura, contrario a lo informado por Block y col (1998) y Jepsen y col (2002), quienes indican que las suturas absorbibles tipo Cat Gut son de prematura absorción, provocando la pérdida del radiotransmisor o mortalidad del individuo.

En esta etapa, el anestesista comienza a alternar las dosis de anestesia con agua filtrada, para dar inicio a la recuperación del individuo. Finalmente, el individuo es llevado al estanque de recuperación donde se mantiene en observación constante, hasta evidenciar su total recuperación anestésica, la cual dura aproximadamente 10 minutos.

Procedimiento y cuidados postoperatorios. Los peces operados se mantienen en recuperación individual en acuarios con condiciones idénticas al periodo de aclimatación, durante un periodo aproximado de 72 horas, como tiempo promedio de espera antes de la liberación del individuo. Se debe tener especial cuidado con las condiciones de velocidad, circulación y temperatura del agua, adecuadas para cada especie según sus requerimientos de hábitat. En esta etapa los parámetros físicos del agua deben ser medidos constantemente para evitar el riesgo de mortalidad. Para ello se mantienen instrumentos de medición continua en cada estanque, midiendo niveles de oxígeno disuelto y temperatura (Sakaris y Jesien 2005). Para la especie D. camposensis estos parámetros deben estar en torno a $10 \mathrm{mg} / \mathrm{L}$ oxígeno disuelto y $12{ }^{\circ} \mathrm{C}$, respectivamente.

Durante este periodo se previno la aparición de cuadros infecciosos alternando diariamente baños de Solflox ${ }^{\circledR}$ (solución antibiótica al 0,015\%) de una hora y la aplicación de productos desinfectantes de uso tópico, a través de curaciones, en las que se evaluó la cicatrización de las incisiones. Cuando fue necesario, según grado de infección e inflamación, se repitió el baño de antibiótico, hasta la completa recuperación del pez. En este sentido, existen tratamientos con antibióticos, los cuales son usados previo y durante la cirugía. Uno de éstos es el uso de oxitetraciclina $(75 \mathrm{mg} / \mathrm{kg}$ del peso del pez) directamente en la incisión (Schulz 2003) o inyectarla directamente en la cavidad celómica, inmediatamente después de haber realizado la cirugía (Daugherty y Sutton 2005). Una vez que las heridas están completamente cicatrizadas y no hay indicios de procesos infecciosos, los peces son liberados en el mismo lugar donde fueron capturados. Al respecto cabe mencionar que durante el procedimiento realizado no se presentaron efectos colaterales no deseados asociados a la cirugía y anestesia, lo cual se puede asociar a los adecuados y metódicos cuidados postoperatorios dados por el equipo de trabajo.

La cirugía para implantes de radiotransmisores en peces es nueva en Chile y para llevarla a cabo se han tenido en consideración todas las variables para evitar las infecciones descritas para siluriformes (Daugherty y Sutton 2005, Sakaris y Jesien 2005, Schulz 2003, Schulz y Leuchtenberger 2006). Los procedimientos aquí descritos resultaron exitosos, logrando descartar la mortalidad postoperatoria, y se realizó un efectivo seguimiento de los peces durante el tiempo de vida útil de los radiotransmisores. Radiotracking es el procedimiento de seguimiento de los individuos marcados, registrando la posición geográfica detalladamente; cada registro de la posición de un individuo corresponde a una lectura, que en este estudio fueron realizadas tres veces por semana para cada individuo. En el momento de ser devueltos a su hábitat original, el individuo busca un refugio y, finalmente, estabiliza los desplazamientos propios de una reaclimatación a su medio.

En general, los desplazamientos de D. camposensis ocurrieron en el sentido de la corriente del río, pero también resultaron importantes los desplazamientos aguas arriba y los recorridos entre una ribera y otra. De este modo, los mayores trayectos registrados fueron de 553,8 m aguas abajo y $248,3 \mathrm{~m}$ aguas arriba. El máximo desplazamiento desde una ribera a otra fue de $420 \mathrm{~m}$, registrado para un pez liberado en la desembocadura del Lago Riñihue.

\section{RESUMEN}

Una metodología para el estudio del comportamiento, patrones de desplazamiento y ámbito de hogar de peces es el seguimiento de individuos que portan radiotransmisores implantados quirúrgicamente. Estudios de aplicación de esta técnica no se han reportado para los peces de agua dulce nativos de Chile. Por lo tanto, no hay protocolos que se hayan desarrollado en Chile para describir los procedimientos de cirugía para la inserción de los implantes ni las dosis de anestésicos y medicamentos aplicados para evitar la mortalidad de los peces. El siguiente trabajo describe el procedimiento realizado para el implante de radiotransmisores en individuos de Diplomystes camposensis, un pez nativo del sur de Chile, que puede ser valioso para la investigación futura. Este procedimiento fue un éxito en estos organismos, ya que impidió la mortalidad postquirúrgica y permitió el seguimiento de peces en toda la vida del transmisor. Este documento sirve como guía para futuros estudios que implican implantes quirúrgicos de transmisores de radio en peces nativos, con alto valor de conservación, en el que la mortalidad debida a la investigación debería ser mínima.

\section{AGRADECIMIENTOS}

Agradecemos las sugerencias de procedimientos efectuadas por Beatriz Muñoz y Daniela Reyes. A Néstor Ortiz, Roberto Cifuentes y Priscila Piedra por colaborar en la captura de los peces, y a Jorge Jiménez por su colaboración en el manuscrito final. Este estudio fue financiado por Colbún S.A.

\section{REFERENCIAS}

Adams NS, DW Rondorf, SD Evans, J E Kelly, RW Perry. 1998. Effects of surgically and gastrically implanted radio transmitters on swimming performance and predator avoidance of juvenile chinook salmon (Oncorhynchus tshawytscha). Can J Fish Aquat Sci 55, 781-787. 
Arratia G. 1983. Preferencias de hábitat de peces siluriformes de aguas continentales de Chile (Fam. Diplomystidae y Trichomycteridae). Stud Neotrop Fauna E 18, 217-237.

Arratia G. 1987. Description of the primitive Family Diplomystidae (Siluriformes, Teleostei, Pisces): morphology, taxonomy and phylogenetic implications. Bonner Zool Monogr 24, 5-120.

Baras E. 1991. A bibliography on underwater telemetry, 1956-1990. Can Tech Rep Fish Aquat Sci 1819, 1-55.

Baras E, C Birtles, L Westerloppe, X Thoreau, M Ovidio, D Jeandrain, JC Philippart. 2003. A critical review of surgery techniques for implanting telemetry devices into the body cavity of fish. In: Le Maho Y (ed). Proceedings of the Fifth European Conference on Wildlife Telemetry, CNRS, Strasbourg, France.

Block BA, H Dewar, T Williams, ED Prince, C Farwell, D Fudge. 1998. Archival tagging of Atlantic bluefin tuna (Thunnus thynnus thynnus). Mar Tech Soc 32, 37-46.

Daugherty DJ, TM Sutton. 2005. Seasonal movement patterns, habitat use, and home range of Flathead Catfish in the Lower St. Joseph River, Michigan. N Am J Fish Manage 25, 256-269.

de Pinna MCC. 1998. Phylogenetic relationships of neotropical Siluriformes (Teleostei: Ostario-physi): historical overview and synthesis of hypotheses. In: Malabarba LR, Reis RE, Vari RP, Lucena ZM, Lucena CA (eds). Phylogeny and Classification of Neotropical Fishes. EDIPUCRS, Porto Alegre, Brazil, Pp 279-330.

Diana JS. 1980. Diel activity pattern and swimming speeds of northern pike (Esox lucius) in Lac Ste. Anne, Alberta. Can J Fish Aquat Sci 37, 2454-2458.

Dyer B. 2000. Systematic review and biogeography of the freshwater fishes of Chile. Estud Oceanol 19, 77-98.

Gallepp GW, JJ Magnuson. 1972. Effects of negative buoyancy on the behavior of the bluegill, Lepomis macrochirus Rafinesque. Trans Am Fish Soc 101, 507-512.

Habit E. 2005. Aspectos de la biología y hábitat de un pez endémico de Chile en peligro de extinción (Diplomystes nahuelbutaensis Arratia, 1987). Interciencia 30, 8-11.

Habit E, A Jara, N Colin, A Oyanedel, P Victoriano, J Gonzalez, K Solis-Lufí. 2008. Threatened fishes of the world: Diplomystes camposensis Arratia, 1987 (Diplomystidae). Environ Biol Fish 84, 393-394.

Jepsen N, A Koed, EB Thorstad, E Baras. 2002. Surgical implantation of telemetry transmitters in fish: how much have we learned? Hydrobiologia 483, 239-248.

Kaseloo PA, AH Weatherly, J Lotimer, MD Farina. 1992. A biotelemetry system recording fish activity. J Fish Biol 40, 165-179.

Lucas MC. 1989. Effects of implanted dummy transmitters on mortality, growth and tissue reaction in rainbow trout, Salmo gairdneri Richardson. J Fish Biol 35, 577-587.

Manns RE, BG Whiteside. 1979. Behavioral variations associated with ultrasonic tagging of Guadalupe bass in Lake Travis, Texas. Underwat Telem Newslett 9, 4-9.
Mesing CL, M Wicker. 1986. Home range, spawning migrations, and homing of radio-tagged Florida largemouth bass in two central Florida lakes. Trans Am Fish Soc 115, 286-295.

Mortensen DG. 1990. Use of staple sutures to close surgical incisions for transmitter implants. In: Parker NC, Giorgi A, Heidinger R, Jester D, Prince E, Winans G (eds). Fish-Marking Techniques. Proceedings International Symposium and Workshop on FishMarking Techniques, American Fisheries Society Symposium, Seattle, USA, Pp 380-383.

Mulcahy DM. 2003. Surgical Implantation of Transmitters into Fish. ILAR Journal 44, 295-306.

Nielsen LA. 1992. Methods of marking fish and shellfish. American Fischeries Society Publication, Bethesda, Maryland, USA.

Paukert CP, PJ Chvala, BL Heikes, ML Brown. 2001. Effects of implanted transmitter size and surgery on survival, growth, and wound healing of bluegill. Trans Am Fish Soc 130, 975-980.

Ruiz VH, M Marchant. 2004. Ictiofauna de aguas continentales chilenas. Ediciones Universidad de Concepción, Concepción, Chile.

Sakaris PC, RV Jesien. 2005. Retention of surgically implanted ultrasonic transmitters in the brown bullhead catfish. N Am J Fish Manage $25,822-826$

Schramm HL, DJ Black. 1984. Anaesthesia and surgical procedures for implanting radio transmitters into grass carp. Prog Fish Cult 46, 185-190.

Schulz UH. 2003. Effects of surgically implanted dummy transmitters on the south american catfish jundiá (Rhamdia quelen). Braz J Biol 63, 345-348.

Schulz U, C Leuchtenberger. 2006. Activity patterns of south american silver catfish (Rhamdia quelen). Braz J Biol 66, 565-574.

Stasko AB, DE Pincock. 1977. Review of underwater biotelemetry with emphasis on ultrasonic techniques. J Fish Res Bd Can 34, 1261-1285.

Summerfelt RC, LS Smith. 1990. Anesthesia, surgery, and related techniques. In: Schreck CB, Moyle PB (eds). Methods for Fish Biology. American Fisheries Society, Bethesda, Maryland, USA, Pp 213-272.

Thoreau X, E Baras. 1997. Evaluation of surgery procedures for implanting telemetry transmitters into the body cavity of blue tilapia Oreochromis aureus. Aquat Living Resour 10, 207-211.

Vila I, M Contreras, L Fuentes. 1996. Reproducción de Diplomystes nahuelbutaensis Arratia 1987 (Pisces: Diplomystidae). Gayana Oceanología 4, 129-137.

Vila I, L Fuentes, M Contreras. 1999. Peces Límnicos de Chile. Boletín Museo Historia Natural de Chile 48, 61-75.

Wagner GN, ED Stevens. 2000. Effects of different surgical techniques: Suture material and location of incision site on the behaviour of rainbow trout (Oncorhynchus mykiss). Mar Fresh Behav Physiol 33, 103-114.

Winter JD. 1996. Advances in Underwater biotelemetry. In: Murphy BR, Willis DW (eds). Fisheries Techniques. $2^{\text {nd }}$ ed. American Fisheries Society Bethesda Maryland, USA, Pp 555-590. 